ARTICLE

\title{
Management of $\mathrm{HIV}$-associated cryptococcal disease in South Africa
}

\author{
N P Govender, ${ }^{1}$ MB BCh, MMed (Micro), FCPath (SA) Micro, MSc , DTM\&H, Dip HIV Man (SA); \\ S Dlamini, ${ }^{2} \mathrm{MB}$ ChB, FCP (SA), Cert ID (SA) \\ ${ }^{1}$ National Institute for Communicable Diseases (Centre for Opportunistic, Tropical and Hospital Infections), Division of the National Health \\ Laboratory Service and Faculty of Health Sciences, University of the Witwatersrand, Johannesburg, South Africa \\ ${ }^{2}$ Division of Infectious Diseases and HIV Medicine, Department of Medicine, Faculty of Health Sciences, University of Cape Town, South Africa
}

Corresponding author: N P Govender (neleshg@nicd.ac.za)

In routine-care settings, the 10 -week mortality associated with cryptococcal meningitis (CM) is high, even with prompt, appropriate antifungal treatment and correctly timed initiation of antiretroviral therapy (ART). While early diagnosis of HIV infection and initiation of ART prior to the development of AIDS is the most important way to reduce the incidence of CM, a cryptococcal antigenaemia screen-and-treat intervention has the potential to reduce mortality by identifying patients prior to onset of CM. Antifungal treatment for HIV-associated CM is divided into three phases over a minimum period of 1 year: $(i)$ a 2-week induction phase, including intravenous amphotericin B deoxycholate as a backbone; (ii) an 8-week consolidation phase with fluconazole 400 mg daily; and (iii) a maintenance phase with fluconazole $200 \mathrm{mg}$ daily. Amphotericin B should be paired with another antifungal agent to maximise cerebrospinal fluid fungal clearance. World Health Organization guidelines emphasise that patients receiving amphotericin B-containing regimens should have access to a 'minimum package of toxicity prevention, monitoring and management to minimise the serious amphotericin B-related toxicities particularly hypokalaemia and nephrotoxicity'. Raised intracranial pressure is a serious and often fatal complication of CM, which requires good pressure management with repeat lumbar punctures. ART should be initiated 4 - 6 weeks after starting antifungal therapy. In many cases, relapse CM among South African patients occurs because of suboptimal adherence to secondary prophylaxis with fluconazole and/or the antifungal not being prescribed.

S Afr Med J 2014;104(12):896. DOI:10.7196/SAMJ.9070

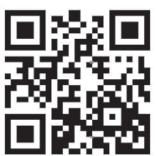

It is appropriate that management of HIV-associated cryptococcal meningitis $(\mathrm{CM})$ is included as a topic in this edition of CME because the opportunistic fungal pathogen, Cryptococcus neoformans, remains the most common cause of adult meningitis in southern Africa. ${ }^{[1,2]}$ In developed countries, widespread access to antiretroviral therapy (ART) during the 1990s was associated with a concomitant decline in the incidence of CM. ${ }^{[3,4]}$ However, the large HIV epidemic in South Africa (SA), coupled with the delayed introduction of ART into the public sector for patients with advanced HIV disease and defaulting of ART, has led to a relatively smaller impact of ART on the incidence of CM. ${ }^{[5,6]}$ The incidence of laboratory-confirmed disease in Gauteng Province decreased from 24 to 16 reported cases of CM per 100000 persons in the general population during 2006 2013. ${ }^{[6]}$ However, this is still higher than the 2002 baseline incidence of 15 reported cases of CM per 100000 persons (N P Govender unpublished data). Furthermore, there have been important recent developments. A simple cryptococcal antigen (CrAg) lateral flow assay (LFA) is now commercially available for more accurate, rapid and potentially point-of-care diagnosis of $\mathrm{CM}$ and asymptomatic antigenaemia. ${ }^{[7,8]}$ New information is also available on a CrAg screenand-treat intervention to prevent CM-related deaths, including costeffectiveness data. ${ }^{[9]}$ Recently published clinical trials have highlighted the most efficacious antifungal agents for induction-phase treatment and optimal timing of ART after a first diagnosis of CM. ${ }^{[10,11]}$

\section{Prevention of CM}

In routine-care settings in sub-Saharan Africa, the 10-week mortality associated with CM is high, even with prompt, appropriate antifungal treatment and correctly timed initiation of ART. CM accounted for 13 - 18\% of all deaths in four Ugandan cohorts of HIV-infected persons and 44\% of deaths in a cohort of SA miners. ${ }^{[12-16]}$ Although it is well recognised that early diagnosis of HIV infection and initiation of ART before the development of AIDS is the most important way to prevent CM, only half of patients in sub-Saharan Africa know their HIV infection status and only $32 \%$ are on ART. ${ }^{[17]}$ Furthermore, a large proportion of patients present to HIV care late. ${ }^{[18,19]}$ Consequently, two focused strategies have been explored to prevent CM among those at highest risk of disease. Primary antifungal prophylaxis is not routinely recommended for patients with a $\mathrm{CD} 4^{+}$count $<100$ cells $/ \mu \mathrm{L}$ unless a prolonged delay in ART initiation is anticipated. ${ }^{[20]}$ An alternative is a screen-and-treat intervention for patients with a $\mathrm{CD} 4^{+}$count $<100$ cells $/ \mu \mathrm{L}$, using CrAg screening of serum or plasma followed by targeted pre-emptive fluconazole therapy for those testing CrAgpositive, provided they do not have CM; this is currently being implemented in SA. ${ }^{[21]}$ The latter intervention is feasible because asymptomatic persons with subclinical cryptococcal disease can be detected from 3 weeks to more than 3 months prior to the development of symptomatic CM, using a simple blood test. ${ }^{[12]}$ The screen-and-treat intervention has been shown to be cost-effective in several settings. ${ }^{[9,22]}$ Based on recent estimates, approximately $2 \%$ of asymptomatic SA persons with a $\mathrm{CD}^{+}$count $<100$ cells $/ \mu \mathrm{L}$ and no prior diagnosis of CM have cryptococcal antigenaemia. ${ }^{[23]}$ The updated Southern African HIV Clinicians Society (SAHIVCS) guidelines recommend that $\mathrm{HIV}$-infected ART-naive adults with a $\mathrm{CD} 4{ }^{+}$count $<100$ cells $/ \mu \mathrm{L}$ should be screened for cryptococcal disease before ART is started and screening for cryptococcal antigenaemia should occur by reflex laboratory or clinician-initiated testing (Fig. 1). ${ }^{[2]}$ 


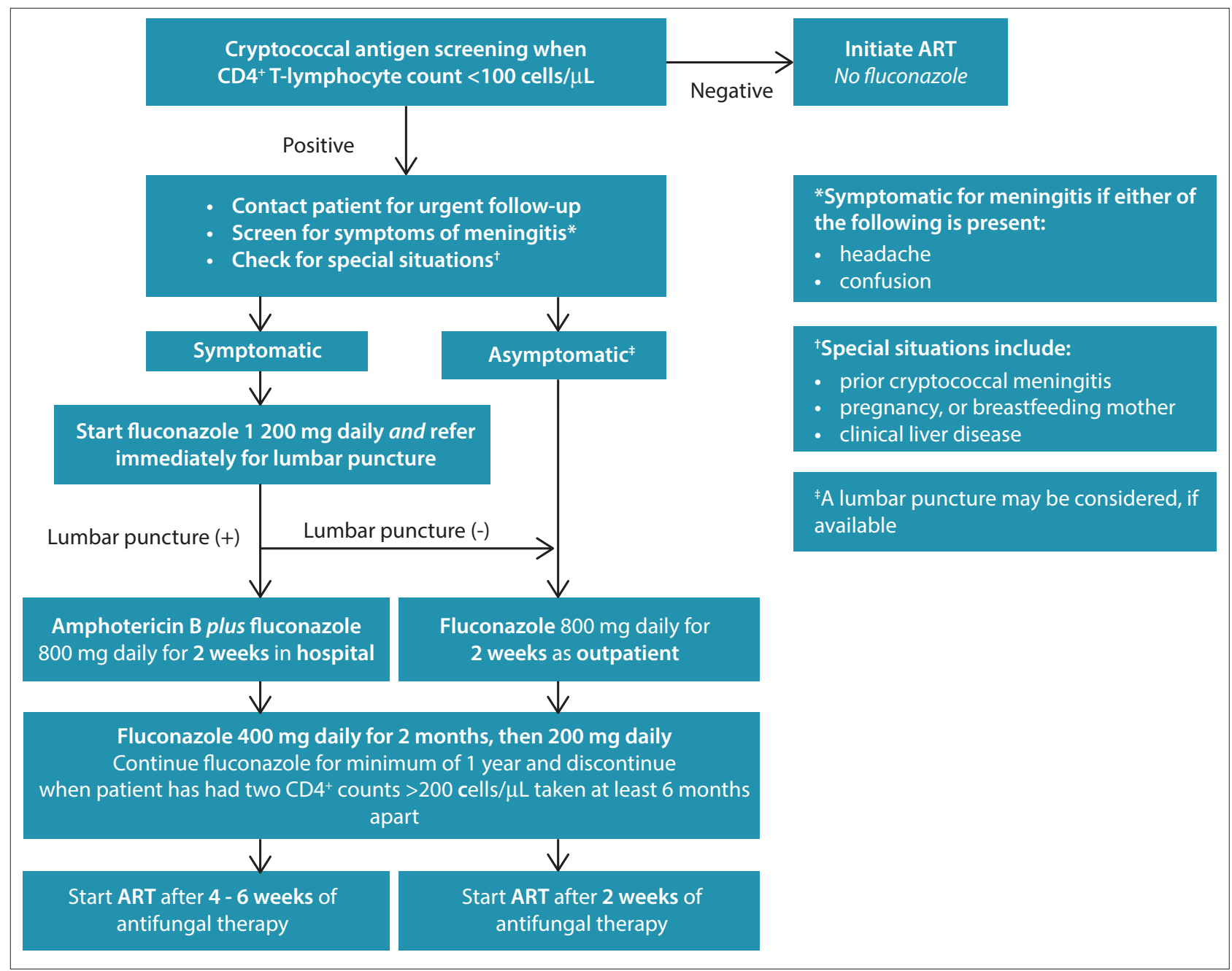

Fig. 1. Cryptococcal screen-and-treat algorithm for antiretroviral therapy (ART)-naive adult patients with a $C D 4^{+} T$-lymphocyte count $<100$ cells/ $/ \mathrm{L}$ (Govender et al. ${ }^{[24)}$.

Patients with a prior diagnosis of CM need not be screened. Those with a new positive CrAg blood test have disseminated cryptococcal disease and should be specifically evaluated for symptoms/signs of meningitis, i.e. headache and confusion. Patients with symptoms/ signs should be referred for lumbar puncture (LP) to exclude CM. If CM is confirmed on LP, they should be managed in hospital (for at least 2 weeks) and ART deferred for 4 - 6 weeks. CrAg-positive patients without symptoms/signs may be offered an LP, if this is immediately accessible, to exclude subclinical meningitis. For $\mathrm{CrAg}$ positive patients without suspected meningitis, oral fluconazole ( $800 \mathrm{mg}$ for 2 weeks, followed by standard consolidation and maintenance treatment) is recommended, also for patients with an LP that is cryptococcal test negative. For those without symptoms/ signs or evidence of meningitis, ART should be started 2 weeks after antifungal therapy is initiated.

\section{Antifungal treatment for CM}

By convention, antifungal treatment for HIV-associated CM among non-pregnant adults is divided into three phases over a minimum period of 1 year: a 2-week induction phase, including intravenous amphotericin B deoxycholate $1 \mathrm{mg} / \mathrm{kg} / \mathrm{d}$ as a backbone; an 8 -week consolidation phase with fluconazole $400 \mathrm{mg} / \mathrm{d}$; and a maintenance phase with fluconazole $200 \mathrm{mg} / \mathrm{d}$ (until the $\mathrm{CD}^{+}$count is $>200$ cells $/ \mu$ l for 6 months on ART and the HIV-1 viral load is suppressed). ${ }^{[24]}$ The fungicidal agent amphotericin B should be paired with another antifungal agent to maximise cerebrospinal fluid (CSF) fungal clearance during the induction phase (Table 1). A recently published Phase III three-arm randomised controlled trial confirmed that the combination of conventional amphotericin B and oral flucytosine for 2 weeks was associated with a $39 \%$ reduction in 10-week mortality and more rapid CSF fungal clearance than amphotericin B monotherapy among HIV-infected patients with CM. ${ }^{[10]}$ However, registration of flucytosine with the South African Medicines Control Council has not been maintained, the cost of the imported medicine is currently prohibitive and this agent is not included in the South African Essential Medicines List for hospitals. ${ }^{[25]}$ In line with guidelines from the World Health Organization (WHO), SAHIVCS guidelines recommend that amphotericin B be paired with high-dose oral fluconazole $(800 \mathrm{mg} / \mathrm{d})$ during the 2 -week induction phase. ${ }^{[20,24]}$ Although fluconazole is fungistatic and less effective in combination than flucytosine, this recommendation is made for two reasons. Firstly, data from a Phase III trial show superior CSF fungal clearance for the combination of amphotericin $B$ and fluconazole than for amphotericin B monotherapy, and data from two trials show a non-significant reduction in mortality and neurological morbidity endpoints. ${ }^{[10,26]}$ Secondly, fluconazole is well tolerated and widely accessible to SA clinicians for the management of CM through the long-standing Diflucan Partnership 
Table 1. Management of an initial episode of cryptococcal meningitis among HIV-infected ART-naive adults

\begin{tabular}{|c|c|}
\hline Diagnosis & $\begin{array}{l}\text { - Confirm the diagnosis by LP and submit cerebrospinal fluid for a rapid test (either } \\
\text { cryptococcal antigen or India ink test) and fungal culture }\end{array}$ \\
\hline Raised intracranial pressure & $\begin{array}{l}\text { - Measure the opening pressure at the time of diagnostic } \mathrm{LP} \text { (in the left lateral supine position) } \\
\text { - If the opening pressure is }>25 \mathrm{cmH}_{2} \mathrm{O} \text {, remove } 10-30 \mathrm{~mL} \text { cerebrospinal fluid } \\
\text { - Repeat therapeutic LPs whenever the patient has symptoms/signs of raised intracranial } \\
\text { pressure, e.g. headache, vomiting, 6th cranial nerve palsy (note: daily LPs may be required) }\end{array}$ \\
\hline Induction-phase antifungal treatment & - Prescribe amphotericin B deoxycholate $1 \mathrm{mg} / \mathrm{kg} / \mathrm{d}$ and fluconazole $800 \mathrm{mg} / \mathrm{d}$ for 14 days \\
\hline $\begin{array}{l}\text { Prevention and laboratory monitoring of } \\
\text { amphotericin B toxicities }\end{array}$ & $\begin{array}{l}\text { - Infuse } 1 \mathrm{~L} \text { normal saline containing } 1 \text { ampoule of potassium chloride over } 2 \text { hours before } \\
\text { the amphotericin B infusion } \\
\text { - Prescribe twice-daily oral potassium and daily oral magnesium supplementation } \\
\text { - Check creatinine and potassium (and magnesium, if test is available) at baseline and twice } \\
\text { weekly } \\
\text { - Check haemoglobin at baseline and weekly }\end{array}$ \\
\hline Outpatient management & $\begin{array}{l}\text { - Prescribe consolidation-phase treatment (fluconazole } 400 \mathrm{mg} / \mathrm{d} \text { ) } \\
\text { - Ensure that the patient is appropriately counselled regarding the diagnosis and need to } \\
\text { continue long-term fluconazole and start ART } \\
\text { - Refer the patient to an ART clinic }\end{array}$ \\
\hline ART & - Start ART 4 - 6 weeks after initiation of antifungal treatment \\
\hline
\end{tabular}

Programme and government procurement of relatively cheap, generic formulations. The WHO guidelines emphasise that patients receiving amphotericin B-containing induction-phase regimens should have access to a 'minimum package of toxicity prevention, monitoring and management to minimise the serious amphotericin B-related toxicities particularly hypokalaemia and nephrotoxicity. ${ }^{[20]}$ In routine practice, amphotericin B-related toxicities are common. In a recent cross-sectional study at sentinel SA hospitals, $12 \%$ of patients with CM developed anaemia, 39\% hypokalaemia and 30\% nephrotoxicity during the course of induction-phase treatment with amphotericin $\mathrm{B}^{[27]}$ The SAHIVCS guidelines recommend that, among patients without preexisting renal impairment and hyperkalaemia, a litre of normal saline containing an ampoule of potassium chloride should be administered intravenously prior to each daily dose of amphotericin B and that pre-emptive oral potassium chloride and magnesium chloride supplements be prescribed for the duration of the amphotericin B-containing induction phase. The guidelines also recommend baseline and twice-weekly monitoring of serum potassium and creatinine, baseline and weekly monitoring of haemoglobin, daily monitoring of fluid input and output and appropriate management of electrolyte abnormalities for the duration of amphotericin B treatment. Raised intracranial pressure (ICP) should be excluded at the time of diagnosis of an episode of CM and considered during hospital admission if symptoms/signs of raised ICP recur. The latter requires pressure management with regular LPs.

\section{Timing of ART}

Determining the optimal time for starting ART involves a balancing act of its benefits compared with the risk of immune reconstitution inflammatory syndrome (IRIS). ${ }^{[28]}$ In general, delaying ART among patients with low $\mathrm{CD}^{+}$counts is associated with a high mortality. Until recently, there has been very little evidence for the optimal time to start ART after the initiation of antifungal treatment for CM. Previously, most study protocols for clinical trials in SA, Uganda, Malawi and Thailand advocated ART initiation 2 - 6 weeks after starting antifungal therapy. ${ }^{[29]}$ There have been four randomised trials that have tried to answer the question of optimal ART timing.
Three of the trials were underpowered to provide definitive guidance on this issue and yielded very different results. The multi-site randomised controlled Cryptococcal Optimal ART Timing (COAT) trial showed that initiation of ART 1 - 2 weeks $(n=88)$ v. 5 weeks after diagnosis $(n=89)$ was associated with a significantly higher 6-month mortality (hazard ratio for death 1.73 (95\% confidence interval 1.06 - 2.82)). ${ }^{[11]}$ This finding applied to all categories of HIV-infected patients, including those with a $\mathrm{CD} 4^{+}$count $<50$ cells $/ \mu \mathrm{L}$. Therefore, the accepted, considered and safe approach is that ART be introduced 4 - 6 weeks after antifungal therapy is started. ${ }^{[24]}$

\section{Relapse of CM}

A culture-confirmed relapse episode should be treated similarly to an initial episode of CM. The cumulative incidence of a symptomatic relapse following an episode of CM is between $6 \%$ and $23 \%{ }^{[30]}$ The potential reasons for a relapse include IRIS, inadequate secondary fluconazole prophylaxis, raised ICP despite fungal clearance or, uncommonly, fluconazole resistance. It is estimated that at least $50-60 \%$ of patients will relapse without secondary prophylaxis and ART. ${ }^{[31]}$ A study conducted in Cape Town over a 2-year period in 57 patients with CM relapse found that 30 were not taking fluconazole; of these, $53 \%$ were non-adherent to therapy and $47 \%$ were discharged without being prescribed secondary prophylaxis or receiving it at the clinic. ${ }^{[31]}$ These data suggest that major causes of inadequate secondary prophylaxis in SA are suboptimal patient adherence and non-prescription of fluconazole secondary prophylaxis. Improving the knowledge of patients and healthcare providers should help to address these problems. Two forms of cryptococcal IRIS are recognised: paradoxical IRIS and unmasking IRIS. Paradoxical IRIS is defined as the recurrence of symptoms/signs of a previously treated opportunistic infection (OI). ${ }^{[28,32]}$ Paradoxical IRIS occurs in $6-45 \%$ of patients with prior CM. ${ }^{[28]}$ After ART initiation, paradoxical IRIS may occur at a median of $1-2$ months or, in some instances, 8 - 9 months later. ${ }^{[28]}$ In cases of severe or refractory IRIS, corticosteroids may be used (starting dose of prednisone $1 \mathrm{mg} / \mathrm{kg}$ reduced over several weeks) in addition to therapeutic LPs. Raised ICP may be a presenting feature of paradoxical IRIS despite fungal 
clearance ${ }^{[33]}$ Raised ICP is a serious and often fatal complication of $\mathrm{CM}$, which requires good pressure management with repeat LPs. A number of studies have shown that repeated LPs are associated with a decreased risk of acute mortality. ${ }^{[34,35]}$

\section{Discussion}

Despite the availability of ART, CM is associated with a high mortality - even with efficacious and prompt antifungal therapy. The induction-phase regimen should include a combination of amphotericin B and fluconazole. Access to flucytosine is limited because this agent is not registered in SA and only available on a compassionate basis. In conjunction with antifungal therapy, an important aspect of management includes adequate control of raised ICP, achieved by LPs performed daily until pressure has normalised and symptoms/signs have resolved. The administration of amphotericin B deoxycholate may lead to a number of complications, including renal toxicity, hypokalaemia and phlebitis; these need to be managed proactively. Tuberculosis and cryptococcal disease are often comorbid conditions ${ }^{[36]}$ and their simultaneous management poses a challenge in terms of drug burden and drug-drug interactions. Furthermore, relapse following an initial episode is common and should prompt a full investigation to determine and manage the underlying cause. Although early HIV diagnosis and ART initiation before the onset of AIDS are major interventions needed to reduce the incidence of $\mathrm{CM}$, the cryptococcal screen-and-treat intervention has the potential to reduce CM-associated mortality.

Conflict of interest. N P Govender has received honoraria from MSD Pty (Ltd) and Pfizer for speaking engagements and an investigator-initiated research grant from Pfizer for an unrelated surveillance project.

\section{References}

1. Jarvis JN, Meinties G, Williams A, Brown Y, Crede T, Harrison TS. Adult meningitis in a setting of high HIV and TB prevalence: Findings from 4961 suspected cases. BMC Infect Dis 2010;10:67. [http:// dx.doi.org/10.1186/1471-2334-10-67]

2. Marais S, Pepper DJ, Schutz C, Wilkinson RJ, Meintjes G. Presentation and outcome of tuberculous meningitis in a high HIV prevalence setting. PLoS One 2011;6(5):e20077. [http://dx.doi.org/10.1371/ journal.pone.0020077]

3. Mirza SA, Phelan M, Rimland D, et al. The changing epidemiology of cryptococcosis: An update from population-based active surveillance in 2 large metropolitan areas, 1992-2000. Clin Infect Dis 2003;36(6):789-794.

4. Friedman GD, Jeffrey FW, Udaltsova NV, Hurley LB. Cryptococcosis: The 1981-2000 epidemic. Mycoses 2005;48(2):122-125

5. Jarvis JN, Boulle A, Loyse A, et al. High ongoing burden of cryptococcal disease in Africa despite antiretroviral roll out. AIDS 2009;23(9):1182-1183. [http://dx.doi.org/10.1097/ QAD.0b013e32832be0fc]

6. National Institute for Communicable Diseases. GERMS-SA Annual Report 2013. Johannesburg: National Institute for Communicable Diseases, 2013. http://www.nicd.ac.za/assets/files/GERMSSA\%20AR\%202013.pdf (accessed 22 October 2014).

7. Jarvis JN, Percival A, Bauman S, et al. Evaluation of a novel point of care cryptococcal antigen (CRAG) test on serum, plasma and urine from patients with HIV-associated cryptococcal meningitis. Clin test on serum, plasma and urine from patients with HIV-associated
Infect Dis 2011;53:1019-10123. [http://dx.doi.org/10.1093/cid/cir613]

8. Boulware DR, Rolfes MA, Rajasingham R, et al. Multisite validation of cryptococcal antigen lateral flow assay and quantification by laser thermal contrast. Emerg Infect Dis 2014;20(1):45-53. [http:// dx.doi.org/10.3201/eid2001.130906
9. Jarvis JN, Harrison TS, Lawn SD, Meintjes G, Wood R, Cleary S. Cost effectiveness of cryptococcal antigen screening as a strategy to prevent HIV-associated cryptococcal meningitis in South Africa. PLoS One 2013;8(7):e69288. [http://dx.doi.org/10.1371/journal.pone.0069288]

10. Day JN, Chau TT, Wolbers M, et al. Combination antifungal therapy for cryptococcal meningitis. N Engl J Med 2013;368(14):1291-1302. [http://dx.doi.org/ 10.1056/NEJMc1305981]

11. Boulware DR, Meya DB, Muzoora C, et al. Timing of antiretroviral therapy after diagnosis of cryptococcal meningitis. N Engl J Med 2014;370(26):2487-2498. [http://dx.doi.org/10.1056/NEJMoal312884]

12. French N, Gray K, Watera C, et al. Cryptococcal infection in a cohort of HIV-1-infected Ugandan adults. AIDS 2002;16(7):1031-1038

13. Okongo M, Morgan D, Mayanja B, Ross A, Whitworth J. Causes of death in a rural, population-based human immunodeficiency virus type 1 (HIV-1) natural history cohort in Uganda. Int J Epidemio 1998;27(4):698-702

14. Castelnuovo B, Manabe YC, Kiragga A, Kamya M, Easterbrook P, Kambugu A. Cause-specific mortality and the contribution of immune reconstitution inflammatory syndrome in the first 3 years after antiretroviral therapy initiation in an urban African cohort. Clin Infect Dis 2009;49(6):965-972. [http://dx.doi.org/10.1086/605500]

15. Kambugu A, Meya DB, Rhein J, et al. Outcomes of cryptococcal meningitis in Uganda before and after the availability of highly active antiretroviral therapy. Clin Infect Dis 2008;46(11):1694-1701. [http:// dx.doi.org/10.1086/587667]

16. Corbett EL, Churchyard GJ, Charalambos S, et al. Morbidity and mortality in South African gold miners: Impact of untreated disease due to human immunodeficiency virus. Clin Infect Dis 2002;34(9):1251-1258.

17. UNAIDS. Gap Report. Geneva: UNAIDS, 2014. http://www.unaids.org/en/media/unaids/contentassets/ documents/unaidspublication/2014/UNAIDS_Gap_report_en.pdf (accessed 22 October 2014).

18. Kigozi IM, Dobkin LM, Martin JN, et al. Late-disease stage at presentation to an HIV clinic in the era of free antiretroviral therapy in Sub-Saharan Africa. J Acquir Immune Defic Syndr 2009;52(2):280-289. [http://dx.doi.org/10.1097/QAI.0b013e3181ab6eab]

19. Agaba PA, Meloni ST, Sule HM, et al. Patients who present late to HIV care and associated risk factors in Nigeria. HIV Med 2014;15(7):396-405. [http://dx.doi.org/10.1111/hiv.12125]

20. World Health Organization. Rapid Advice - Diagnosis, Prevention and Management of Cryptococcal Disease in HIV-infected Adults, Adolescents and Children. Geneva: World Health Organization, 2011 http://www.who.int/hiv/pub/cryptococcal_disease2011/en/(accessed 22 October 2014).

21. Govender NP, Roy M, Oladoyinbo S, et al. Phased implementation of screening for cryptococcal disease in South Africa. S Afr Med J 2012;102(12):914-917. [http://dx.doi.org/10.7196/samj.6228]

22. Meya DB, Manabe YC, Castelnuovo B, et al. Cost-effectiveness of serum cryptococcal antigen screening to prevent deaths among HIV-infected persons with a CD4+ cell count $\leq 100$ cells $/ \mu \mathrm{L}$ who start HIV therapy in resource-limited settings. Clin Infect Dis 2010;51:448-455. [http://dx.doi. org/10.1086/655143]

23. Govender N, Chetty V, Spencer D, et al. Cryptococcal screening in Gauteng province, South Africa: Update from the first year of implementation, 2012-2013 (oral abstract 52). Southern African HIV Clinicians Society conference. Cape Town, South Africa, September 2014.

24. Govender NP, Meintijes G, Bicanic T, et al. Guideline for prevention, diagnosis and management of cryptococcal meningitis among HIV-infected persons: 2013 update. S Afr J HIV Med 2013;14(2):76-86.

25. Govender N, Meinties G, Banoo S. Access to flucytosine for HIV-infected patients with cryptococcal meningitis - an urgent need. S Afr Med J 2014;104(9):594-595. [http://dx.doi.org/10.7196/SAMI.8713]

26. Pappas PG, Chetchotisakd P, Larsen RA, et al. A phase II randomized trial of amphotericin B alone or combined with fluconazole in the treatment of HIV-associated cryptococcal meningitis. Clin Infect Dis 2009;48(12):1775-1783. [http://dx.doi.org/10.1086/599112]

27. Meiring S, Fortuin-de Smidt M, Sibiya L, Kularatne R, Dawood H, Govender NP. Prevalence and management of amphotericin B deoxycholate-related toxicities during treatment of HIV-associated cryptococcal meningitis at South African hospitals (oral abstract). Federation of Infectious Disease Societies of Southern Africa conference. Drakensberg, South Africa, October 2013.

28. Locieties of Southern Africa conference. Drakensberg, South Africa, October 2013 . Longley N, Harrison TS, Jarvis JN. Cryptococcal immune reconstitution inflammatory sy
Curr Opin Infect Dis 2013;26(1):26-34. [http://dx.doi.org/10.1097/QCO.0b013e32835c21d1]

29. Jarvis JN, Bicanic T, Loyse A, et al. Determinants of mortality in a combined cohort of 501 patients arvis JN, Bicanic T, Loyse A, et al. Determinants of mortality in a combined cohort of 501 patients
with HIV-associated cryptococcal meningitis: Implications for improving outcomes. Clin Infect Dis with HIV-associated cryptococcal meningitis: Implications
2014;58(5):736-745. [http://dx.doi.org/10.1093/cid/cit794]

30. Musubire AK, Boulware DR, Meya DB, Rhein J. Diagnosis and management of cryptococcal relapse. AIDS Clin Res 2013;Suppl 3(3):S3-003.

31. Jarvis JN, Meintjes G, Williams Z, Rebe K, Harrison TS. Symptomatic relapse of HIV-associated cryptococcal meningitis in South Africa: The role of inadequate secondary prophylaxis. S Afr Med cryptococcal meningitis

32. Bahr N, Boulware DR, Marais S, Scriven J, Wilkinson RJ, Meinties G. Central nervous system immune reconstitution inflammatory syndrome. Curr Infect Dis Rep 2013;15(6):583-593. [http://dx.doi. org/10.1007/s11908-013-0378-5]

33. Cinti SK, Armstrong WS, Kauffman CA. Case report. Recurrence of increased intracranial pressure with antiretroviral therapy in an AIDS patient with cryptococcal meningitis. Mycoses 2001;44(1112):497-501.

34. Rolfes MA, Hullsiek KH, Rhein J, et al. The effect of therapeutic lumbar punctures on acute mortality from cryptococcal meningitis. Clin Infect Dis 2014;ciu596. [Epub ahead of print]

35. Bicanic T, Brouwer AE, Meintjes G, et al. Relationship of cerebrospinal fluid pressure, fungal burden and outcome in patients with cryptococcal meningitis undergoing serial lumbar punctures. AIDS 2009:23(6):701-706. [http://dx.doi.org/10.1097/QAD.0b013e32832605fe]

36. Rawat D, Capoor MR, Nair D, Deb M, Aggarwal P. Concomitant TB and cryptococcosis in HIVinfected patients. Trop Doct 2008;38(4):251-252. [http://dx.doi.org/10.1258/td.2007.070295] 